\title{
Oak Ridge Research Reactor Quarterly Report January, February, and March of 1976
}

\author{
S. S. Hurt, III \\ E. D. Lance
}




\section{DISCLAIMER}

This report was prepared as an account of work sponsored by an agency of the United States Government. Neither the United States Government nor any agency Thereof, nor any of their employees, makes any warranty, express or implied, or assumes any legal liability or responsibility for the accuracy, completeness, or usefulness of any information, apparatus, product, or process disclosed, or represents that its use would not infringe privately owned rights. Reference herein to any specific commercial product, process, or service by trade name, trademark, manufacturer, or otherwise does not necessarily constitute or imply its endorsement, recommendation, or favoring by the United States Government or any agency thereof. The views and opinions of authors expressed herein do not necessarily state or reflect those of the United States Government or any agency thereof. 


\section{DISCLAIMER}

Portions of this document may be illegible in electronic image products. Images are produced from the best available original document. 
Printed in the United States of America: Available from National Technical Information Service

U.S. Department of Commerce

5285 Port Royal Road, Springfield, Virginia 22161

Price: Printed Copy $\$ 4.00$; Microfiche $\$ 2.25$

This report was prepared as an account of work sponsored by the United States Government. Neither the United States nor the Energy Research and Development Administration/United States Nuclear Regulatory Commission, nor any of their employees, nor any of their contractors, subcontractors, or their employees, makes any warranty, express or implied, or assumes any legal liability or responsibility for the accuracy, completeness or usefulness of any information, apparatus, product or process disclosed, or represents that its use would not infringe privately owned rights. 
Contract No. W-7405-eng-26

OPERATIONS DIVISION

OAK RIDGE RESEARCH REACTOR QUARTERLY REPORT

JANUARY, FEBRUARY, AND MARCH OF 1976

Date Published: October 1976

S, S. Hurt, III

E. D. Lance

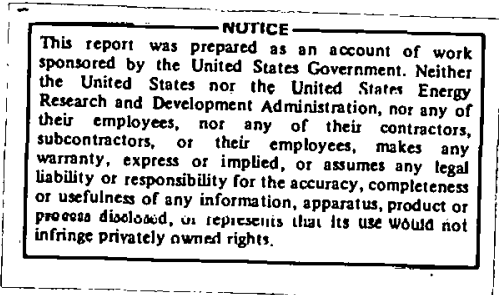

NOTICE This document contains information of a preliminary nature and was prepared primarily for internal use at the Oak Ridge National Laboratory, It is subjoct to rovision or corrootion and theroforo dooc not represent a final report.

OAK RIDGE NATIONAL LABORATORY

Oak Ridge, Tennessee 37830 operated by UNION CARBIDE CORPORATION

for the

ENERGY RESEARCH AND DEVELOPMENT ADMINISTRATION 


\section{THIS PAGE \\ WAS INTENTIONALLY \\ LEFT BLANK}


CONTENTS

PAGE

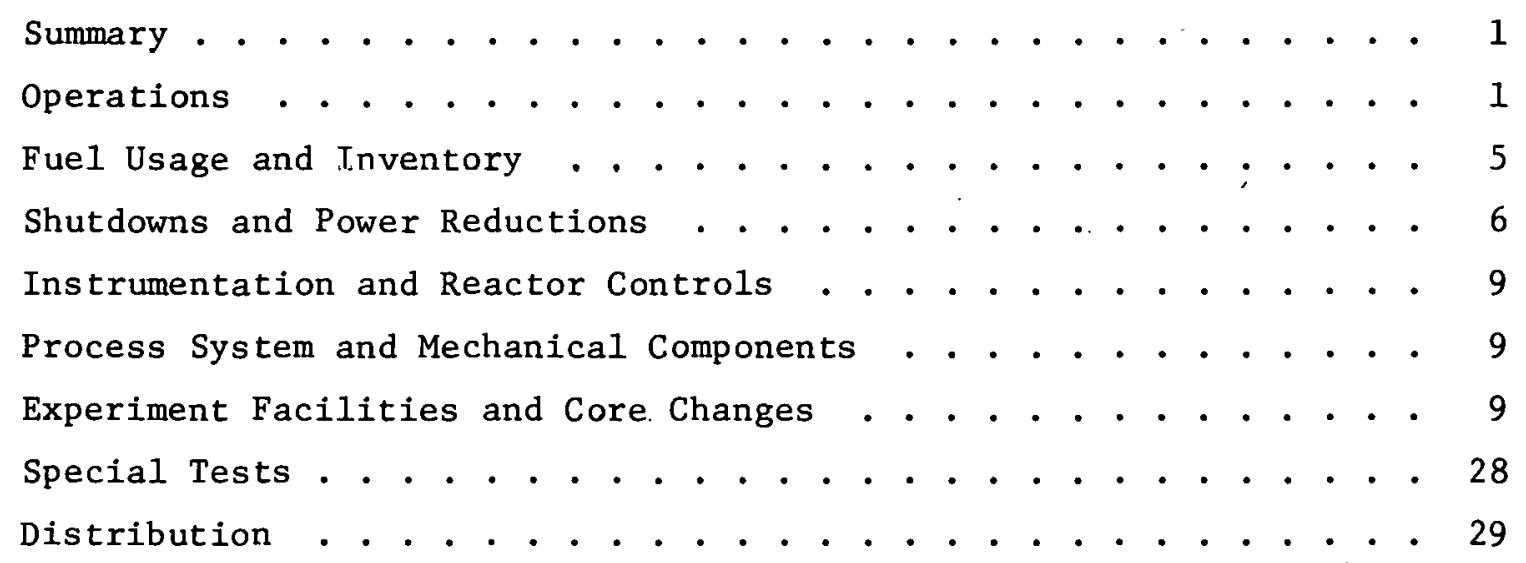


SUMMARY

The ORR operated at an average power level of $29.9 \mathrm{Mw}$ for $82.8 \%$ of the time during January, February, and March of 1976. Two fuel elements were declared spent (58.8\% average burnup) during the quarter, while twenty-eight new elements were placed in service. Four shim-rod elements were retired from service at an average burnup of $64.4 \%$.

The reactor was shutdown on nine occasions, one of which was unscheduled. Two end-of-cycle shutdowns accounted for $92.1 \%$ of the total downtime. The remaining downtime was used principally for refueling and experiment work.

Maintenance activities, both mechanical and instrument, were essentially routine in nature.

Special tests or measurements completed during the quarter were three calorimeter gamma heating runs made in CP-E5 during the end of Cycle 129 shutdown.

\section{OPERATIONS}

The basic operating data presented in Table 1 indicates that the ORR operation for the quarter was normal as compared with recent experience.

Details relative to cycles of operation during the quarter are given in Table 2, while Figures 1 and 2 are plots of shim-rod position versus time for these cycles. 
Table 1. ORR Basic Operating Data January, February, and March of 1976

\begin{tabular}{|c|c|c|c|c|}
\hline & $\begin{array}{l}\text { This } \\
\text { Quarter }\end{array}$ & $\begin{array}{c}\text { Last } \\
\text { Quarter }\end{array}$ & $\begin{array}{l}\text { Year } \\
\text { To Date }\end{array}$ & $\begin{array}{l}\text { January - } \\
\text { March, } 1975\end{array}$ \\
\hline Total energy, Mwd & $2,254.5$ & $2,333.4$ & $2,254.4$ & $2,238.2$ \\
\hline $\begin{array}{l}\text { Average power, } \\
\text { Mw/operating hr }\end{array}$ & 29.9 & 29.9 & 29.9 & 29.7 \\
\hline Time operating, $\%$ & 82.8 & 84.9 & 82.8 & 84.9 \\
\hline Availability, \% & 90.6 & 86.7 & 90.6 & 89.0 \\
\hline $\begin{array}{l}\text { Reactor water radio- } \\
\text { activity } \frac{\mathrm{c} / \mathrm{min}}{\mathrm{ml}}(\mathrm{av})\end{array}$ & 28,200 & 27,600 & 28,200 & 30,600 \\
\hline $\begin{array}{l}\text { Pool water radio- } \\
\text { activity } \frac{\mathrm{c} / \mathrm{min}}{\mathrm{ml}} \text { (av) }\end{array}$ & 610 & 640 & 610 & 690 \\
\hline $\begin{array}{l}\text { Reactor water resis- } \\
\text { tivity, ohm-cm (av) }\end{array}$ & 822,000 & $1,050,000$ & 822,000 & 812,000 \\
\hline $\begin{array}{l}\text { Pool water resis- } \\
\text { tivity, ohm-cm (av) }\end{array}$ & 799,000 & 761,000 & 799,000 & 818,000 \\
\hline Fuel elements depleted & 2 & 11 & 2 & 33 \\
\hline $\begin{array}{l}\text { Average burnup of fuel } \\
\text { elements depleted, } \%\end{array}$ & 58.8 & 52.5 & 58.8 & 52.6 \\
\hline Shim rods depleted & 4 & 4 & 4 & 4 \\
\hline $\begin{array}{l}\text { Average burnup of shim } \\
\text { rods depleted, } \%\end{array}$ & 64.4 & 63.0 & 64.4 & 64.6 \\
\hline Radioisotope samples & 0 & 1 & 0 & 2 \\
\hline Research samples & 1 & 8 & 1 & 5 \\
\hline
\end{tabular}

Table 2. Cycles of Operation

\begin{tabular}{llcc}
\hline Cycle No. & Date Begun & Date Ended & $\begin{array}{c}\text { Accumulated } \\
\text { Energy (Mwd) }\end{array}$ \\
\hline $129 \cdot$ & December 11, 1975 & January 25, 1976 & $1,302.7^{*}$ \\
130 & January 29,1976 & - March 21, 1976 & $1,520.2$
\end{tabular}

*734.2 Mwd this report period. 


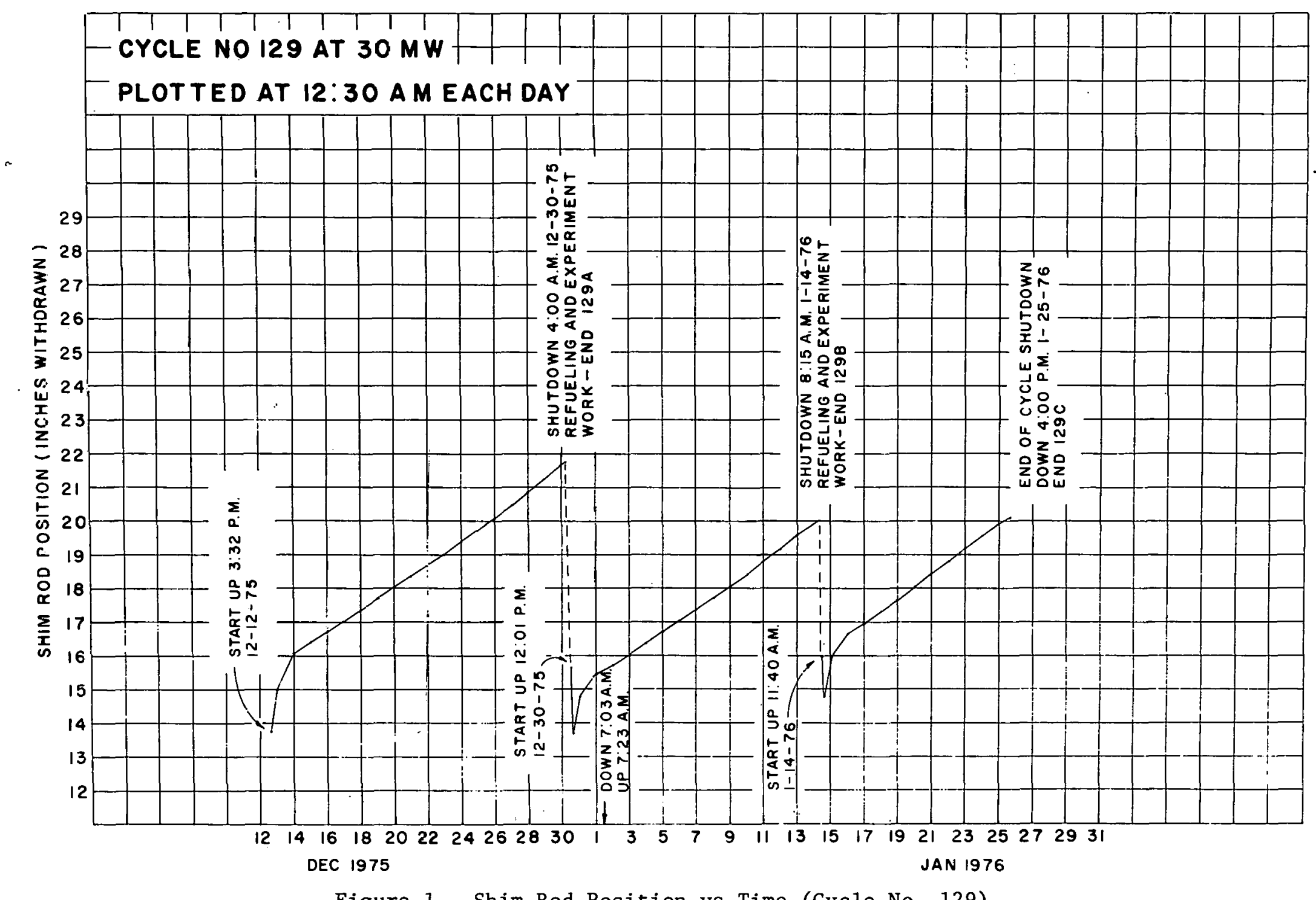

$\omega$ 


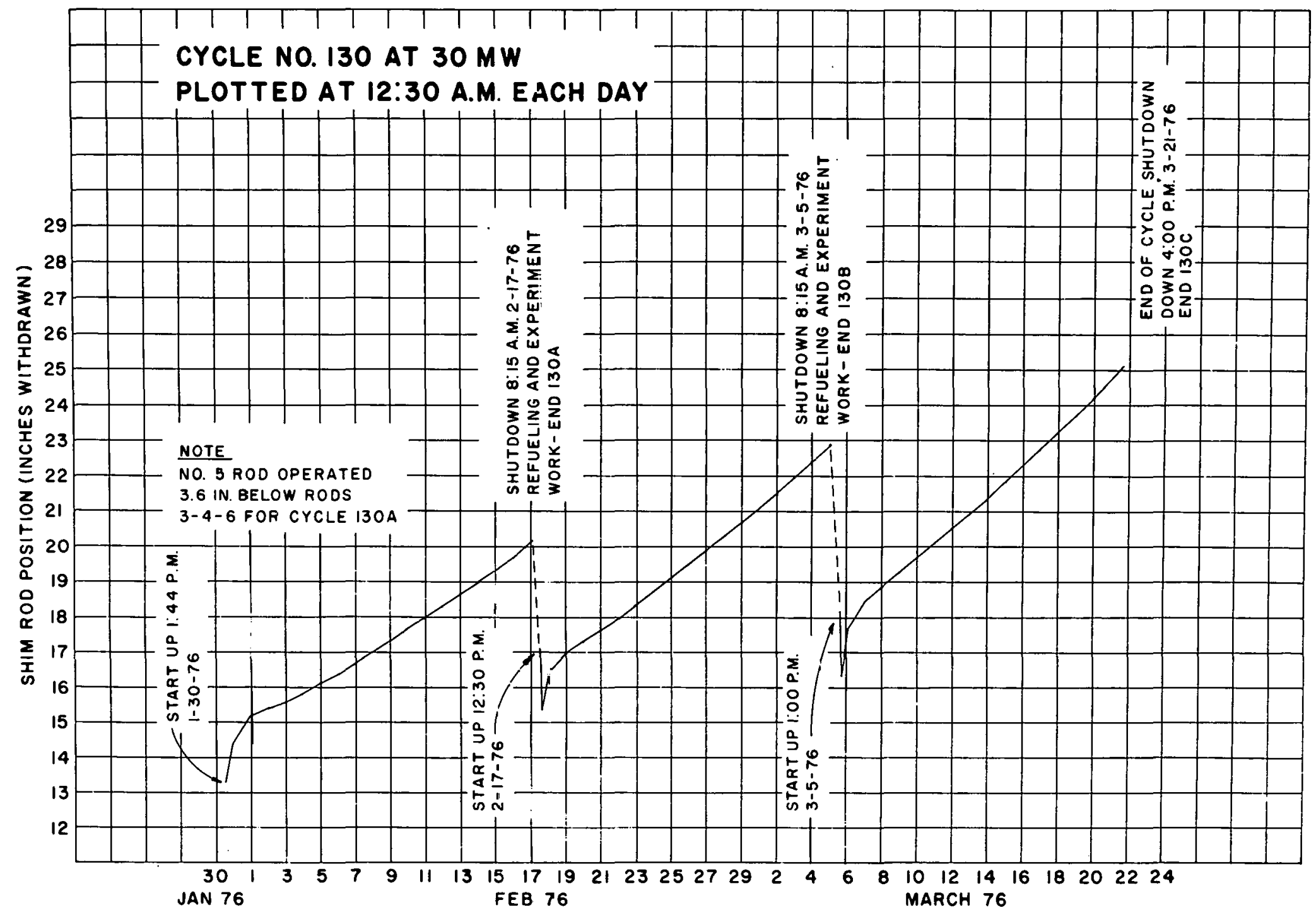

Figure 2. Shim Rod Position vs Time (Cycle No. 130) 
FUEL USAGE AND INVENTORY

Twenty-eight new fuel elements were placed in service during the quarter. Only two fuel elements were declared spent during the quarter due to the lowering of the minimum fuel content from 120 grams to 100 grams before declaring an element spent. Forty-five fuel elements and six shimrod elements were transferred to the Savannah River Plant for chemical separation.

Other details of fuel usage and inventory may be found in Tables 1 and 3 .

Table 3. ORR Fue1 Status

\begin{tabular}{|c|c|c|c|c|}
\hline & $\begin{array}{l}\text { This } \\
\text { Quarter }\end{array}$ & $\begin{array}{l}\text { Last } \\
\text { Quarter }\end{array}$ & $\begin{array}{c}\text { Jan-Mar } \\
1976\end{array}$ & $\begin{array}{c}\text { Jan-Mar } \\
1975\end{array}$ \\
\hline $\begin{array}{l}\text { Depleted fuel elements trans- } \\
\text { ferred for chemical separa- } \\
\text { tion }\end{array}$ & 45 & 17 & 45 & 59 \\
\hline $\begin{array}{l}\text { Average percent burnup of fuel } \\
\text { elements transferred }\end{array}$ & 52.0 & 53.0 & 52.0 & 48.1 \\
\hline $\begin{array}{l}\text { New fuel elements placed in } \\
\text { service }\end{array}$ & 28 & 29 & 28 & 16 \\
\hline $\begin{array}{l}\text { New fuel elements available } \\
\text { for use }\end{array}$ & $93.0^{*}$ & 63 & & \\
\hline $\begin{array}{l}\text { Depleted shim rods transferred } \\
\text { for chemical separation }\end{array}$ & 6 & 0 & 6 & 12 \\
\hline $\begin{array}{l}\text { Average percent burnup of shim } \\
\text { rods transferred }\end{array}$ & 65.0 & --- & 65.0 & 65.0 \\
\hline New shim rods placed in service & 4 & 4 & 4 & 4 \\
\hline New shim rods available for use & 0 & 0 & 0 & 0 \\
\hline
\end{tabular}


SHUTDOWNS AND POWER REDUCTIONS

Reactor downtime (power level $<\mathrm{N}_{\mathrm{L}}$ ) totaled approximately 375 hours. A summary of the shutdowns is given in Table 4, and details of each are contalned in Table 5. Table 6 offers information relative to the unscheduled shutdown.

Table 4. Summary of Shutdowns

\begin{tabular}{|c|c|c|}
\hline Description of Shutdown & Number & Downtime (hr) \\
\hline \multicolumn{3}{|c|}{ Scheduled } \\
\hline Regular, end of cycle & 2 & 345.550 \\
\hline $\begin{array}{l}\text { Regular: refueling, and } \\
\text { iodine removal }\end{array}$ & 3 & 12.450 \\
\hline $\begin{array}{l}\text { Special: end of gamma heat } \\
\text { measurement run at } \mathrm{N}_{L}\end{array}$ & 2 & 0.784 \\
\hline $\begin{array}{l}\text { Special: end of gamma heat } \\
\text { measurement run at } N_{L} \text {, and } \\
\text { completion of end-of-cycle } \\
\text { shutdown }\end{array}$ & 1 & 16.017 \\
\hline Sub total & 8 & 374.801 \\
\hline \multicolumn{3}{|c|}{ Unscheduled } \\
\hline Human error & 1 & 0.333 \\
\hline Subtotal & 1 & 0.333 \\
\hline Total & 9 & 375.134 \\
\hline
\end{tabular}


Table 5. ORR Scheduled Shutdowns, Details

\begin{tabular}{|c|c|c|c|}
\hline Date & $\begin{array}{l}\text { Duration } \\
\text { (hr) }\end{array}$ & $\begin{array}{l}\text { End } \\
\text { Cycle }\end{array}$ & Remarks \\
\hline $1-14-75$ & 3.417 & $129 \mathrm{~B}$ & $\begin{array}{l}\text { The reactor was refueled and isotope (iodine, xenon) work was } \\
\text { completed. }\end{array}$ \\
\hline $1-25-76$ & 97.550 & $129 \mathrm{C}$ & $\begin{array}{l}\text { This shutdown marked the end of cycle } 129 \text {. Shutdown activities } \\
\text { included: (1) The replacement of the water seals on the } \\
\text { reactor secondary water pump No. } 1 \text {, (2) the removal of a faulty } \\
\text { pressure gauge on the secondary side of the reactor heat ex- } \\
\text { changers Nos. } 1 \text { and } 2 \text {, ( } 3 \text { ) the removal and installation of an } \\
\text { experiment in core position } B-8,(4) \text { the removal and install- } \\
\text { ation of an experiment in poolside } P-5 \text {, and (5) the removal } \\
\text { and insertion of an experiment in poolside } P-4 \mathrm{~A} \text {. }\end{array}$ \\
\hline $1-29-76$ & 0.417 & & $\begin{array}{l}\text { The shutdown terminated a low power }\left(\mathrm{N}_{L}\right) \text { run for gamma heat } \\
\text { measurement in core position } \mathrm{E}-5 \text {. }\end{array}$ \\
\hline $1-29-76$ & 0.367 & & $\begin{array}{l}\text { The shutdown terminated a low power }\left(\mathrm{N}_{L}\right) \text { run for gamma heat } \\
\text { measurement in core position } E-5 \text {. }\end{array}$ \\
\hline $1-29-76$ & 16.017 & & $\begin{array}{l}\text { This shutdown ended a low power }\left(\mathrm{N}_{\mathrm{L}}\right) \text { run for gamma heat measure- } \\
\text { ment in core position } \mathrm{E}-5 \text {. The end-of-cycle } 129 \text { shutdown work } \\
\text { was completed. This consisted of ( } 1 \text { ) the replacement of the } \\
\text { bypass filters in the west filter columns on the reactor pri- } \\
\text { mary water system, and (2) the routine programmed maintenance. }\end{array}$ \\
\hline $2-17-76$ & 4.283 & $130 \mathrm{~A}$ & $\begin{array}{l}\text { The reactor was refueled and isotope (iodine, xenon) work was } \\
\text { completed. }\end{array}$ \\
\hline
\end{tabular}


Iable 5 (continued)

\begin{tabular}{|c|c|c|c|}
\hline Date & $\begin{array}{l}\text { Duration } \\
\quad(h r)\end{array}$ & $\begin{array}{l}\text { End } \\
\text { Cycle }\end{array}$ & Remarks \\
\hline $3-5-76$ & 4.750 & $130 \mathrm{~B}$ & $\begin{array}{l}\text { The reactor was refueled and isotope (iodine, xenon) work was } \\
\text { completed. }\end{array}$ \\
\hline $3-21-76$ & 248.000 & $130 \mathrm{C}$ & $\begin{array}{l}\text { This shutdown marked the end of cycle } 130 \text {. Shutdown activities } \\
\text { included: (1) The cleaning of the primary side of the pool } \\
\text { heat exchanger, (2) the installation of a new oil seal on the } \\
\text { pool cooling fan gear reducer, (3) the replacement of the } \\
\text { No. } 1 \text { counting rate fission chamber, (4) the wiring changes in } \\
\text { ORR Reactor Control (Change Memo No. 112), made to limit the } \\
\text { setjack in reactor power to } 0.6 \mathrm{~N}_{\mathrm{F}} \text { for the new experiment OC-1, } \\
\text { and (5) the installation of the new experiment OC-1 in CP-E5. } \\
\text { One experiment was removed from poolside P-4A and one was } \\
\text { removed from poolside P-5. }\end{array}$ \\
\hline
\end{tabular}

Table 6. ORE Unscheduled Shutdowns, Details

\begin{tabular}{cccc}
\hline Date & $\begin{array}{c}\text { Duration } \\
(\mathrm{hr})\end{array}$ & $\begin{array}{c}\text { End } \\
\text { Cycle }\end{array}$ & Remarks \\
\hline $1-1-76$ & 0.333 & $\begin{array}{c}\text { The selected No. } 1 \text { log-N amplifier was inadvertenly turned to } \\
\text { "Ground" setting when checking out troubles in the No. } 2 \\
\text { log-N channel. }\end{array}$ \\
\hline
\end{tabular}


INSTRUMENTATION AND REACTOR CONTROLS

The performance of the instrumentation for the complex was quite satisfactory and only rather routine maintenance was required as indicated in Table 7. Table 8 reflects the status of the various fission and ionization chambers.

PROCESS SYSTEM AND MECHANICAL COMPONENTS

Details of maintenance or changes to the process system are given in Table 9. Table 10 summarizes the results of efficiency test of the various gaseous waste filters. There were no repairs or changes related to the reactor mechanical components (drives, bearings, etc.).

EXPERIMENT FACILITIES AND CORE CHANGES

Experiment facility usage is given in Table 11. The core configuration used during the quarter is shown in Figure No. 3. 
For Fuel Cycles 129 B, C, 130 A, B, C,

\begin{tabular}{|c|c|c|c|c|c|c|c|c|}
\hline 1 & 2 & 3 & 4 & 5 & 6 & 7 & 8 & 9 \\
\hline $\mathrm{Be}$ & $\mathrm{Be}$ & $I^{131}$ & $F$ & $\mathrm{~F}$ & $F$ & $\mathrm{~F}$ & $F$ & $\mathrm{Be}$ \\
\hline $\mathrm{Be}$ & $\mathrm{Be}$ & $F$ & $S$ & $\mathrm{~F}$ & $S$ & $F$ & $E$ & $\mathrm{Be}$ \\
\hline $\mathrm{Be}$ & $\mathrm{Be}$ & $F$ & $F$ & $F$ & $F$ & $F$ & $\mathrm{~F}$ & $\mathrm{Be}$ \\
\hline $\mathrm{Be}$ & $\mathrm{F}$ & F & $S$ & $\mathrm{~F}$ & S & $\mathbf{F}$ & HT & $\mathrm{Be}$ \\
\hline $\mathrm{Be}$ & $\mathrm{F}$ & $E$ & $\mathrm{~F}$ & $\mathrm{~F}$ & $\mathrm{~F}$ & $\mathrm{E}$ & $\mathrm{F}$ & $\mathrm{Be}$ \\
\hline $\mathrm{Be}$ & $\mathrm{F}$ & $F$ & $S$ & $\mathrm{~F}$ & S & $\mathrm{F}$ & $\mathbf{F}$ & $\mathrm{Be}$ \\
\hline $\mathrm{Be}$ & $\mathrm{Be}$ & $\mathrm{Be}$ & $\mathrm{Be}$ & $\mathrm{Be}$ & $\mathrm{Be}$ & $\mathrm{Be}$ & $\mathrm{Be}$ & $\mathrm{Be}$ \\
\hline
\end{tabular}

LATTICE COMPONEN'I'

Fue1

Shim Rod (SR)

Beryllium (Be)

Experiment (E)

Iodine Sleeve $\left({ }^{131} I\right)$

Isotope Stringer (Iso)

Hydraulic Tube (HT)
NUMBER

28

$-6$

-_24

3

$1 . . . .$.

$\underline{0}$

1

Figure 3. ORR Lattice Configuration - January 1, 1976 through March 21, 1976. 
Table 7. Maintenance and Changes, Instrumentation and Controls

\begin{tabular}{lll}
\hline Date & Component & Trouble or Change \\
\hline
\end{tabular}

No. 1 Counting-Rate Channel

No, 1 Counting- Inoperative
rate chamber $\begin{gathered}\text { The fission chamber was replaced with } \\ \text { chamber TSM-1-F52 (IC-8138) which } \\ \text { corrected the problem. }\end{gathered}$

No. 2 Counting-Rate Channel

2-4-76 No. 2 Counting- Replacement

rate meter

The counting-rate meter was replaced to correct an erratic readout.

No. 1 Jog-N Channel

2-24-76 Log-N records

Negative spikes on readout

The recorder slidewire and contacts were cleaned.

No. 2 Log-N Channel

1-2-76 Amplifier. No power supply

-

The sola transformer that feeds power to No. $2 \log -\mathrm{N}$ channel was replaced.

No. 1 Gamma Channe1

1-21-76 No. 1 Gamma recorder
Inoperative chart drive motor
The chart drive motor was replaced. 
Table 7 (continued)

\begin{tabular}{llll}
\hline Date & Component & Trouble or Change & Reason or Maintenance \\
\hline
\end{tabular}

No. 2 Gamma Channel

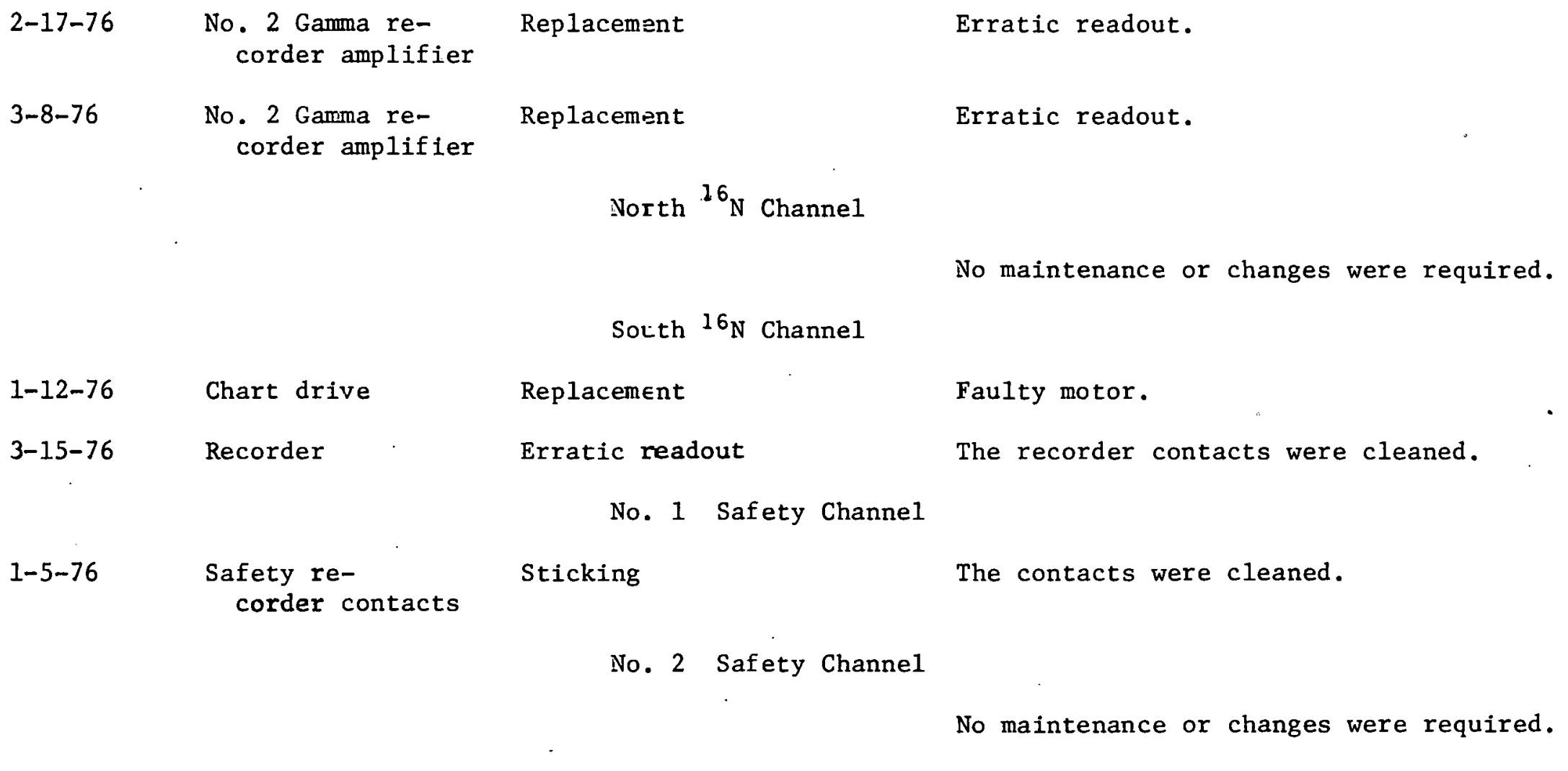

No. 3 Safety Channe1

No maintenance or changes were required. 
Table 7 (continued)

\begin{tabular}{|c|c|c|c|}
\hline Date & Component & Trouble or Change & Reason or Maintenance \\
\hline \multicolumn{4}{|c|}{ Magnet Amplifiers } \\
\hline $2-17-76$ & $\begin{array}{c}\text { No. 1A Magnet } \\
\text { amplifier }\end{array}$ & $\begin{array}{l}\text { Fluctuating magnet } \\
\text { current }\end{array}$ & Replacement of electronic tubes. \\
\hline $2-17-76$ & $\begin{array}{l}\text { No. 1B Magnet } \\
\text { amplifier }\end{array}$ & $\begin{array}{l}\text { F1uctuating magnet } \\
\text { current }\end{array}$ & The amplifier was replaced. \\
\hline \multicolumn{4}{|c|}{ Micromicroammeter Channel } \\
\hline
\end{tabular}

No maintenance or changes were required.

Servo Channel

No maintenance or changes were required.

Process Instrumentation

$\begin{array}{lll}2-17-76 & \begin{array}{l}\text { No. } 1 \Delta T \\ \text { recorder battery }\end{array} & \text { Replacement } \\ & \begin{array}{l}\text { No. } 2 \Delta \mathrm{T} \\ \text { recorder battery }\end{array} & \text { Replacement } \\ & \begin{array}{l}\text { No. } 1 \text { Outlet tem- } \\ \text { perature recorder }\end{array} & \text { Replacement } \\ & \text { battery } & \\ & \begin{array}{l}\text { No. } 2 \text { Outlet tem- } \\ \text { perature recorder } \\ \text { battery }\end{array} & \text { Replacement } \\ & \end{array}$

The battery was replaced to correct an erratic readout.

The battery was replaced to correct an erratic readout.

The battery was replaced to correct an erratic readout.

The battery was replaced to correct an erratic readout. 
Iable 7 (continued)

\begin{tabular}{|c|c|c|c|}
\hline Date & Component & Trouble or Change & Reason or Maintenance \\
\hline \multicolumn{4}{|c|}{ Process Instrumentation } \\
\hline $3-26-76$ & $\begin{array}{l}\text { Pressurized off-gas } \\
\text { set point tempera- } \\
\text { ture element (TM- } \\
\text { OF-2) }\end{array}$ & Replacement & $\begin{array}{l}\text { Indications of a breakdown caused by } \\
\text { the age of the element. }\end{array}$ \\
\hline \multirow[t]{2}{*}{$2-24-76$} & $\begin{array}{c}\text { Reactor secondary } \\
\text { pH - problem }\end{array}$ & Faulty probes & The probes were replaced. \\
\hline & & Raciation Detection & Instrumentation \\
\hline $2-13-76$ & $\begin{array}{l}\text { Reactor secondary } \\
\text { activity channel }\end{array}$ & Faulty probe & The probe was replaced. \\
\hline $3-8-76$ & $\begin{array}{l}\text { Monitrons (reactor } \\
\text { primary pump cell } \\
\text { Nos. } 1 \text { and } 3 \text { ) }\end{array}$ & Tube replacement & $\begin{array}{l}\text { The tubes were replaced to give a } \\
\text { reliable readout. }\end{array}$ \\
\hline $1-9-76$ & $\begin{array}{l}\text { Scintillation } \\
\text { counter }\end{array}$ & Replacement & The obsolete decade scaler was replaced \\
\hline
\end{tabular}


Table 8. Present Status of Ionization Chambers and Fission Chambers

\begin{tabular}{|c|c|c|c|c|c|c|}
\hline $\begin{array}{l}\text { Chamber } \\
\text { Serial No. }\end{array}$ & Location & & $\begin{array}{l}\text { Present } \\
\text { Service }\end{array}$ & $\begin{array}{l}\text { Date Present } \\
\text { Service Started }\end{array}$ & $\begin{array}{l}\text { Previous } \\
\text { Service }\end{array}$ & Remarks \\
\hline \multicolumn{7}{|c|}{ Installed Chambers } \\
\hline $\begin{array}{l}\text { PCP-III- } \\
105-66-9\end{array}$ & Position 7 & $\begin{array}{l}\text { No. } 1 \\
\text { No. } 2\end{array}$ & $\begin{array}{l}1 \text { safety \& } \\
2 \log N\end{array}$ & $\begin{array}{l}9-66 \\
\text { (safety) }\end{array}$ & None & $\begin{array}{l}\text { No trouble; fabricated at ORNL. } \\
\text { Compensated section used from } \\
\text { February, 1969, until March, } \\
1970 \text {, as No. } 1 \text { log N. Used } \\
\text { as No. } 2 \text { log N from May 28, } \\
1973 .\end{array}$ \\
\hline $\begin{array}{l}\text { CTC-2 } \\
(C-768)\end{array}$ & Position 3 & No.: & 3 safety & $12-12-75$ & LITR-BSR & $\begin{array}{l}\text { Served as LITR safety chamber } \\
\text { from February, 1963, to July, } \\
1965 \text {. Modified for under- } \\
\text { water service in } 1969 \text {. In- } \\
\text { stalled in the BSR. in April, } \\
1970 \text {, and in August the } \\
\text { chamber was removed to stor- } \\
\text { age. }\end{array}$ \\
\hline $\begin{array}{l}\text { PCP-III- } \\
1 \mathrm{CI} 6 \mathrm{~A} \\
\mathrm{SN}-72-3\end{array}$ & Position 2 & $\begin{array}{l}\text { No. } \\
\text { No. }\end{array}$ & $\begin{array}{l}2 \text { safety \& } \\
1 \log \mathrm{N}\end{array}$ & $5-15-75$ & None & None \\
\hline $\begin{array}{l}\text { PCP-II- } \\
106-66-3\end{array}$ & Position 4 & No. & 3 safety & $\begin{array}{l}5-74 \text { (safety) } \\
3-70 \text { ( } \log \mathrm{N}) \\
\text { removed from } \\
\text { service } 12-12-75\end{array}$ & None & $\begin{array}{l}\text { Fabricated by ORNL. Chamber } \\
\text { failed December 12, } 1975 .\end{array}$ \\
\hline $\begin{array}{l}\text { PCP-II } \\
106-63-4\end{array}$ & Position 5 & $\begin{array}{l}\text { Serv } \\
\mathrm{mi} \\
\mathrm{ami}\end{array}$ & $\begin{array}{l}\text { io and } \\
\text { icromicro- } \\
\text { ameter }\end{array}$ & $6-64$ & None & No trouble; fabricated by ORNL. \\
\hline
\end{tabular}


Table 8 (continued)

\begin{tabular}{|c|c|c|c|c|c|}
\hline $\begin{array}{l}\text { Chamber } \\
\text { Serial No. }\end{array}$ & Location & $\begin{array}{l}\text { Present } \\
\text { Service }\end{array}$ & $\begin{array}{l}\text { Date Present } \\
\text { Service Started }\end{array}$ & $\begin{array}{l}\text { Previous } \\
\text { Service }\end{array}$ & Remarks \\
\hline TSM-1-F5 2 & Poolside & $\begin{array}{l}\text { No. } 1 \text { Counting } \\
\text { rate : north }\end{array}$ & $3-29-76$ & ORR & $\begin{array}{l}\text { Used as No. } 2 \text { counting rate } \\
\text { (south) from Apri1 26, 1972, } \\
\text { until October } 24,1972 \text {. } \\
\text { December 5, } 1973 \text { until August } 8, \\
1975 .\end{array}$ \\
\hline $\begin{array}{l}\text { CTC-5 } \\
(\mathrm{C}-770)\end{array}$ & Position 8 & $\begin{array}{l}\text { Chamber discon- } \\
\text { nected }\end{array}$ & $10-71$ & LITR & $\begin{array}{l}\text { Used as No. I safety at the } \\
\text { LITR; modified for ORR-BSR } \\
\text { underwater service. }\end{array}$ \\
\hline $\begin{array}{l}\text { PCP-II- } \\
105-63-2\end{array}$ & $\begin{array}{l}\text { Poolside, } \\
\text { south }\end{array}$ & $\begin{array}{l}\text { No. } 1 \text { gamma, } \\
\text { south }\end{array}$ & $4-26-71$ & ORR & $\begin{array}{l}\text { Used as No. } 2 \text { gamma, north, } \\
\text { from April, 1964, until } \\
\text { December, } 1970 .\end{array}$ \\
\hline $\begin{array}{l}\text { PCP-II } \\
105-63-3\end{array}$ & $\begin{array}{l}\text { Poolside } \\
\text { north }\end{array}$ & $\begin{array}{l}\text { No. } 2 \text { gamma, } \\
\text { north }\end{array}$ & $3-17-71$ & ORR & $\begin{array}{l}\text { Used as No. } 1 \text { gamma, south } \\
\text { from April, } 1964 \text {, until } \\
\text { March, 1970. }\end{array}$ \\
\hline TSM-3-F29 & Poolside & $\begin{array}{l}\text { No. } 2 \text { counting } \\
\text { rate }\end{array}$ & $\Xi 0-24-72$ & None & $\begin{array}{l}\text { Replaced fission chamber "snake" } \\
\text { assembly TSM-1-F52. }\end{array}$ \\
\hline \multicolumn{6}{|c|}{ Spare Chambers } \\
\hline $\begin{array}{l}\text { PCP-II- } \\
125-63-1\end{array}$ & $\begin{array}{l}\text { Instrumen- } \\
\text { tation \& } \\
\text { Controls } \\
\text { Shop }\end{array}$ & Awaiting repair & $4-26-7 \cdot 2$ & ORR & $\begin{array}{l}\text { Used as No. } 1 \text { gamma, south } \\
\text { from March } 31,1970 \text {, until } \\
\text { April 26, } 1972 .\end{array}$ \\
\hline $\begin{array}{l}\text { S } 27-34 R- \\
\text { F29 }\end{array}$ & $\begin{array}{l}\text { ORR plug } \\
\text { storage }\end{array}$ & Awaiting repair & $4-26-71$ & ORR & $\begin{array}{l}\text { Used as No. } 1 \text { counting rate from } \\
\text { July } 28,1970 \text {, to April } 26,1971\end{array}$ \\
\hline
\end{tabular}


Table 8 (continued)

\begin{tabular}{|c|c|c|c|c|c|}
\hline $\begin{array}{l}\text { Chamber } \\
\text { Serial No. }\end{array}$ & Location & $\begin{array}{l}\text { Present } \\
\text { Service }\end{array}$ & $\begin{array}{l}\text { Date Present } \\
\text { Service Started }\end{array}$ & $\begin{array}{l}\text { Previous } \\
\text { Service }\end{array}$ & Remarks \\
\hline$S 3-8 R-F 22$ & $\begin{array}{l}\text { ORR plug } \\
\text { storage }\end{array}$ & Awaiting repair & $4-26-71$ & ORR & $\begin{array}{l}\text { Used as No. } 2 \text { counting rate } \\
\text { from January } 14,1970 \text {, to } \\
\text { Apri1 26, } 1971 .\end{array}$ \\
\hline CTC-1 & $\begin{array}{l}\text { Instrumen- } \\
\text { tation \& } \\
\text { Controls } \\
\text { Shop }\end{array}$ & Awaiting repair & $10-71$ & ORR & $\begin{array}{l}\text { Used as noise monitor from } \\
\text { March, 1962, until October } 1 \text {, } \\
\text { 1971. }\end{array}$ \\
\hline $\begin{array}{l}\text { CTC-4 } \\
(C-769)\end{array}$ & $\begin{array}{l}\text { ORR plug } \\
\text { storage }\end{array}$ & & & LITR & $\begin{array}{l}\text { Used as No. } 2 \text { safety in the } \\
\text { LITR; modified for ORR-BSR } \\
\text { underwater service. }\end{array}$ \\
\hline $\begin{array}{l}\text { PCP-III- } \\
105-66-5\end{array}$ & $\begin{array}{l}\text { Instrumen- } \\
\text { tation \& } \\
\text { Controls } \\
\text { Shop }\end{array}$ & Awaiting repair & . & ORR & $\begin{array}{l}\text { Used as No. } 2 \text { safety and } \log N \\
\text { from September } 27,1968 \text {, to } \\
\text { July } 22,1970 \text {, and from May } 7 \text {, } \\
\text { 1971, to May } 20 \text {, } 1971 \text {; from } \\
\text { November, } 1971 \text {, to January } 24 \text {, } \\
1973 \text {, as No. } 2 \text { safety and unti } \\
\text { May 28, 1973, as No. } 2 \text { log N. }\end{array}$ \\
\hline TSM-2-F45 & $\begin{array}{l}\text { Instrumen- } \\
\text { tation \& } \\
\text { Controls } \\
\text { Shop }\end{array}$ & Awaiting repair & & ORR & $\begin{array}{l}\text { Used as No. } 1 \text { counting rate from } \\
\text { Apri1 } 26,1971 \text {, until December } \\
5,1972 \text {. Removed March } 30 \text {, } \\
1976 \text { to shop for repair. }\end{array}$ \\
\hline
\end{tabular}


Table 9. Process Systems, Maintenance and Changes

\begin{tabular}{|c|c|c|c|c|}
\hline Date & Component & & Remarks & \\
\hline . & Reactor & Primary & Cooling System & \\
\hline $\begin{array}{l}1-1-76 \\
\text { through } \\
1-30-76\end{array}$ & $\begin{array}{l}\text { Primary cooling system in the } \\
\text { primary pump area (discharge } \\
\text { manifold and } 27 \text {-inch line to } \\
\text { the } Y-\text { pit). }\end{array}$ & & $\begin{array}{l}\text { The installation of concrete walls on each side } \\
\text { of the water line was completed; supports were } \\
\text { installed for the line. Boiler plate was placed } \\
\text { over the opening which allows access to the line } \\
\text { for inspection. The pump discharge manifold } \\
\text { inside the pumphouse (Bldg. 3085) was exposed } \\
\text { for inspection in a similar manner. }\end{array}$ & \\
\hline $1-27-76$ & Bypass filters (west column) & & $\begin{array}{l}\text { The filter cartridges were changed. The drain } \\
\text { valve on the column was repaired. }\end{array}$ & $\infty$ \\
\hline $2-19-76$ & $\begin{array}{l}\text { North reactor demineralizer } \\
\text { anion column }\end{array}$ & & $\begin{array}{l}\text { A defective gasket on the bottom of the column } \\
\text { was replaced. }\end{array}$ & \\
\hline $2-23-76$ & South reactor demineralizer & & $\begin{array}{l}\text { A broken shear pin in the lazy rod to valve No. } 27 \\
\text { was replaced. }\end{array}$ & \\
\hline $2-27-76$ & Decay tank & & $\begin{array}{l}\text { An audio inspection of the primary flow through } \\
\text { the tank was performed. No change in noise } \\
\text { from previous inspection was detected. }\end{array}$ & \\
\hline $3-22-76$ & Bypass filters & & The filter cartridges were changed. & \\
\hline $3-24-76$ & Reactor tank access cover & & The Lucite windows and gaskets were replaced. & \\
\hline $3-24-76$ & $\begin{array}{l}\text { Drain line 109-A north of } \\
\text { Bldg. } 3085 \text { (pumphouse) }\end{array}$ & & A section of the drain line was replaced. & \\
\hline , & . & & & \\
\hline > & & r & ' & \\
\hline
\end{tabular}


Table 9 (continued)

\begin{tabular}{lll}
\hline Date & Component & Remarks \\
\hline
\end{tabular}

Reactor Secondary Cooling System

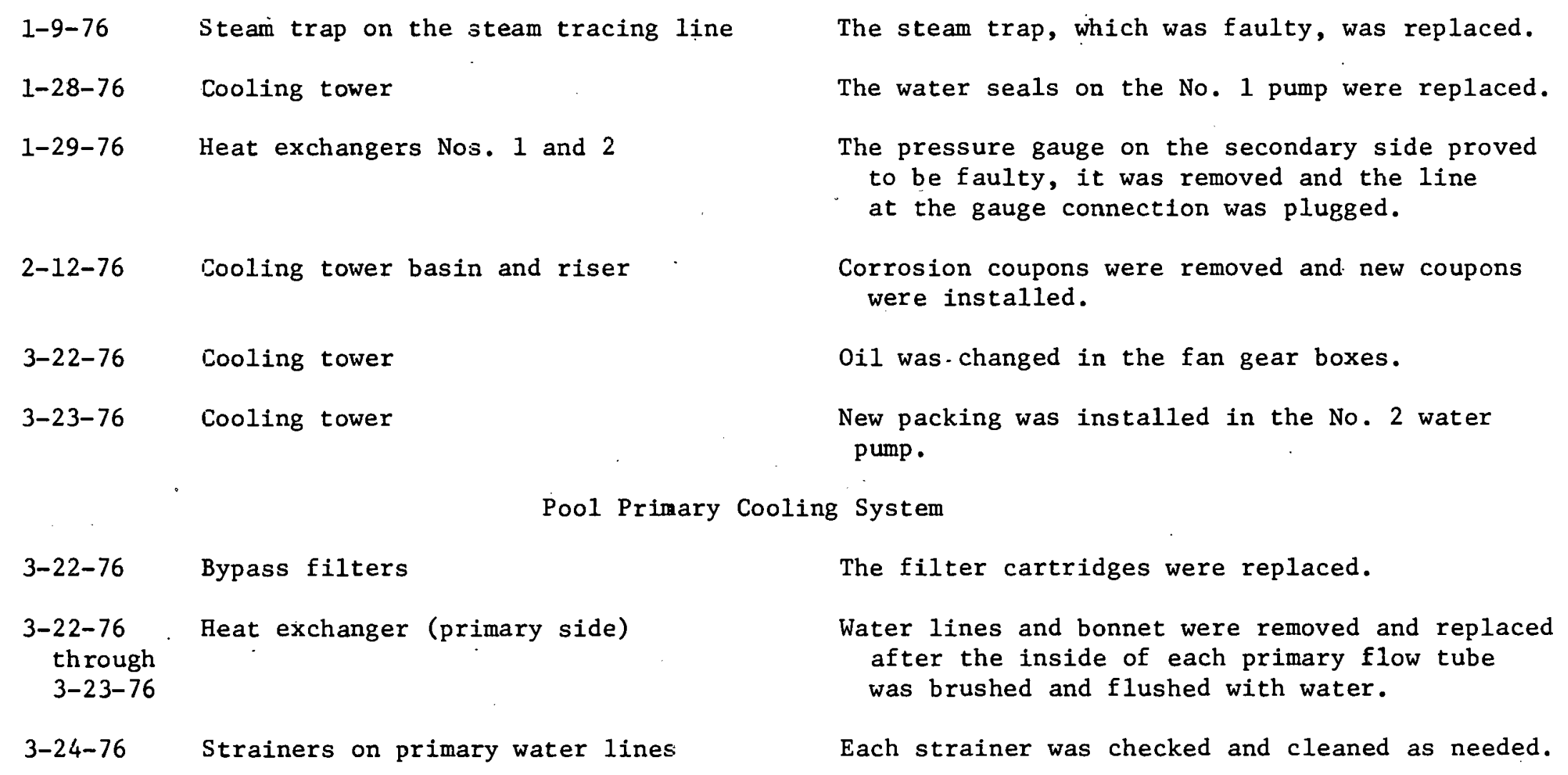


Table 9 (continued)

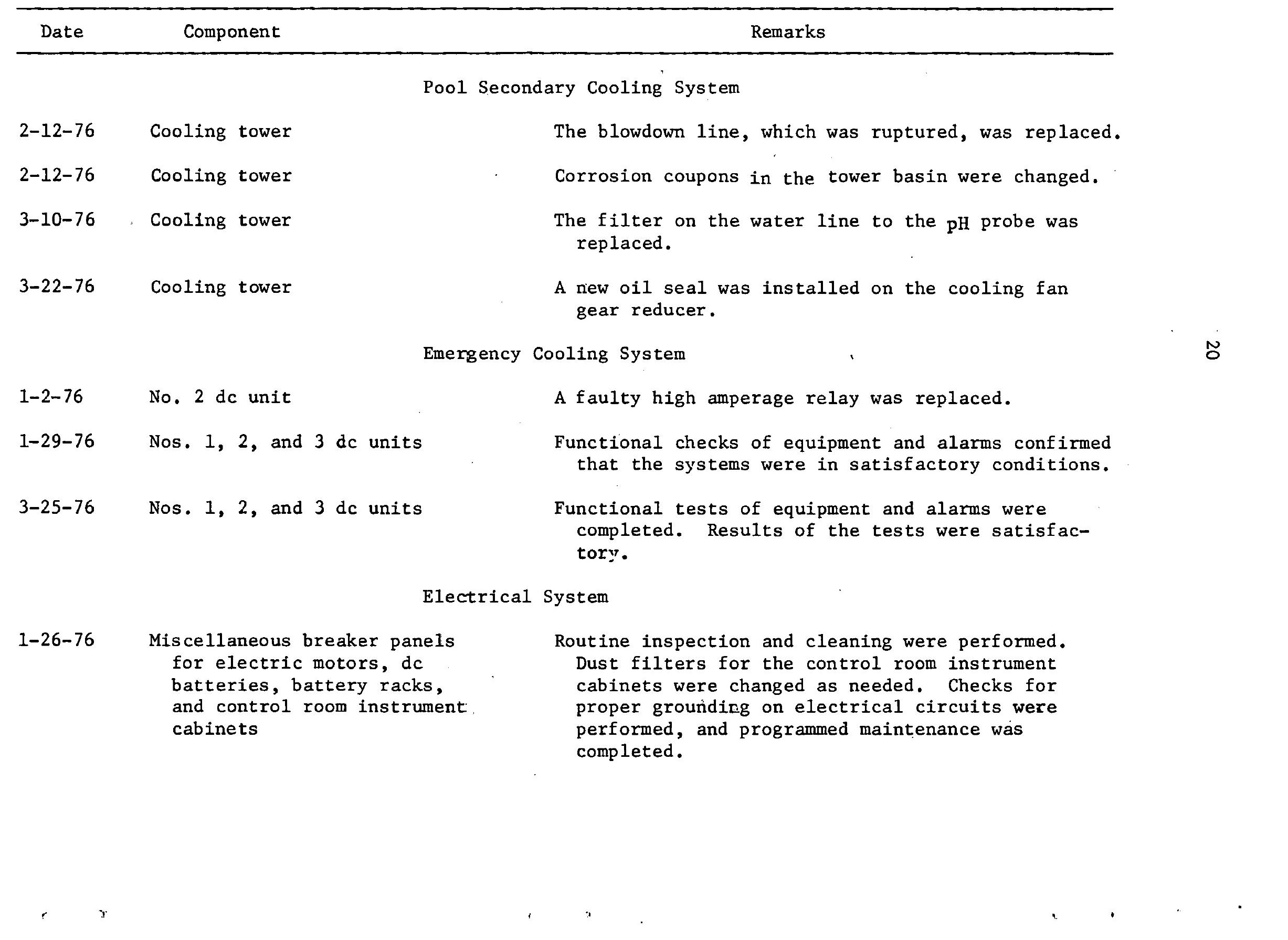


Table 9 (continued)

\begin{tabular}{|c|c|c|}
\hline Date & Component & Remarks \\
\hline \multicolumn{3}{|c|}{ Electrical System } \\
\hline $3-9-76$ & $\begin{array}{l}\text { Reactor pool side electrical } \\
\text { receptacles }\end{array}$ & $\begin{array}{l}\text { The regular electrical receptacles on the pool } \\
\text { parapet were replaced with ground fault } \\
\text { detector receptacles. }\end{array}$ \\
\hline $\begin{array}{l}3-22-76 \\
\text { through } \\
1-26-76\end{array}$ & $\begin{array}{l}\text { Miscallaneous breaker panels for } \\
\text { electric motors, dc batteries, } \\
\text { battery racks, } 2400 \mathrm{~V} \text { transformer } \\
\text { and } 440 \mathrm{~V} \text { breaker at the reactor } \\
\text { secondary cooling tower, and } \\
\text { control room instrument cabinets }\end{array}$ & $\begin{array}{l}\text { Routine inspection and cleaning were performed. } \\
\text { Dust filters for the control room instrument } \\
\text { cabinets were changed as needed. The ac } \\
\text { current on all three reactor primary water } \\
\text { pumps were checked, and programmed main- } \\
\text { tenance was completed. }\end{array}$ \\
\hline \multirow[t]{2}{*}{$\begin{array}{r}3-24-76 \\
\text { through } \\
3-26-76\end{array}$} & $\begin{array}{l}\text { ORR Reactor Control Change Memo. } \\
\text { No. } 112\end{array}$ & $\begin{array}{l}\text { Wiring changes were made to limit the setback } \\
\text { in reactor power to } 0.6 \mathrm{~N} \text { on the new exper- } \\
\text { iment OC-1 that is to be Inserted into the } \\
\text { reactor core position } \mathrm{E}-5 \text { on } 3-29-76 \text {. The } \\
\text { change is for the computer based setbacks only }\end{array}$ \\
\hline & Emergency & Electrical System \\
\hline $1-28-76$ & Diesel generator & $\begin{array}{l}\text { The routine end-of-cycle load test was performed } \\
\text { with satisfactory results. }\end{array}$ \\
\hline $3-23-76$ & Diesel generator & $\begin{array}{l}\text { The routine end-of-cycle load test was performed } \\
\text { with satisfactory results. }\end{array}$ \\
\hline $\begin{array}{l}3-24-76 \\
\vdots\end{array}$ & Wiring for Buildirg 3003 & $\begin{array}{l}\text { Replaced emergency power wiring from the diesel } \\
\text { generator. This was necessary because of an } \\
\text { increase in the emergency power load at } 3003 \text {. }\end{array}$ \\
\hline
\end{tabular}


Table 9 (continued)

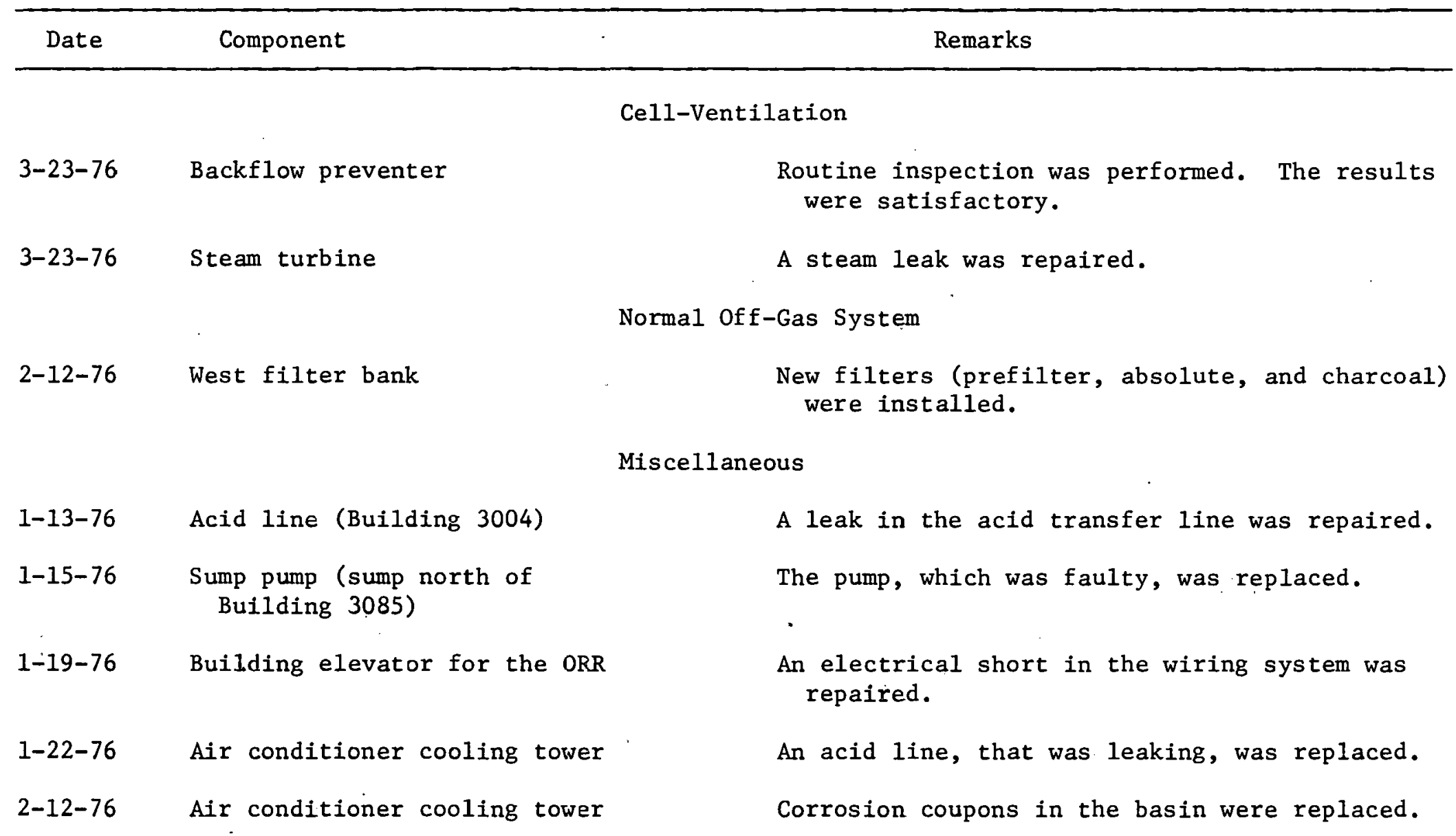


Table 9. (continued)

\begin{tabular}{|c|c|c|}
\hline Date & Component & Remarks \\
\hline \multicolumn{3}{|c|}{ Miscellaneous (continued) } \\
\hline $2-26-76$ & $\begin{array}{l}\text { Condensate pump ir: the southeast } \\
\text { corner of basement in Building } 3042 \\
\text { (ORR) }\end{array}$ & A float switch on the pump was repaired. \\
\hline $3-23 \div 76$ & $\begin{array}{l}\text { Steam line at the pool secondary } \\
\text { pump }\end{array}$ & $\begin{array}{l}\text { A blank cap was installed on a } 125^{\circ} \text { lb steam } \\
\text { line for increased safety. }\end{array}$ \\
\hline $3-23-76$ & Sump pump No. 1 & A broken float arm was repaired. \\
\hline
\end{tabular}


Table 10. Status of Filters - Gaseous Waste Systems

\begin{tabular}{|c|c|c|c|c|c|}
\hline $\begin{array}{l}\text { Type } \\
\text { Filter }\end{array}$ & $\begin{array}{c}\text { Bank } \\
\text { Designation }\end{array}$ & $\begin{array}{c}\text { Date } \\
\text { Installed }\end{array}$ & $\begin{array}{l}\text { Date Last } \\
\text { Tested }\end{array}$ & Type Test & $\begin{array}{c}\text { Retention } \\
\text { Efficiency (\%) }\end{array}$ \\
\hline \multicolumn{6}{|c|}{ Cel1-Ventilation Filters } \\
\hline CWS & Overal1* & $\begin{array}{l}\text { North, 6-20-73 } \\
\text { South, 1-12-65 }\end{array}$ & $3-24-76$ & $\mathrm{DOP}$ & 99.993 \\
\hline Charcoal & Both Banks & $\begin{array}{l}\text { North, } 9-16-74 \\
\text { South, } 10-17-67\end{array}$ & $3-2-76$ & Elemental Iodine & 99.97 \\
\hline Charcoal & Both Banks & $\begin{array}{l}\text { North, } 9-16-74 \\
\text { South, } 10-1 ?-67\end{array}$ & $3-4-76$ & Methy1 Iodide & 34.22 \\
\hline \multicolumn{6}{|c|}{ Pressurizable Off-Gas Filters } \\
\hline $\begin{array}{l}\text { CWS } \\
\text { CWS }\end{array}$ & $\begin{array}{l}\text { North } \\
\text { South }\end{array}$ & $\begin{array}{l}5-5-75 \\
10-18-67\end{array}$ & $\begin{array}{l}3-24-76 \\
3-24-76\end{array}$ & $\begin{array}{l}{ }^{-D O P} \\
\text { DOP }\end{array}$ & $\begin{array}{l}99.965 \\
99.992\end{array}$ \\
\hline $\begin{array}{l}\text { Charcoal } \\
\text { Charcoal }\end{array}$ & $\begin{array}{l}\text { North } \\
\text { South }\end{array}$ & $\begin{array}{l}2-26-73 \\
8-11-75\end{array}$ & $\begin{array}{l}1-28-76 \\
8-13-75\end{array}$ & $\begin{array}{l}\text { Elemental Iodine } \\
\text { Elemental Iodine }\end{array}$ & $\begin{array}{l}99.94 \\
99.993\end{array}$ \\
\hline $\begin{array}{l}\text { Charcoal } \\
\text { Charcoal }\end{array}$ & $\begin{array}{l}\text { North } \\
\text { South }\end{array}$ & $\begin{array}{l}2-26-73 \\
8-11-75\end{array}$ & $\begin{array}{l}10-7-75 \\
10-8-75\end{array}$ & $\begin{array}{l}\text { Methyl Iodide } \\
\text { Methy1 Iodide }\end{array}$ & $\begin{array}{l}87.97 \\
97.48\end{array}$ \\
\hline \multicolumn{6}{|c|}{ Basement Hood Exhaust } \\
\hline $\begin{array}{l}\text { CWS } \\
\text { CWS }\end{array}$ & $\begin{array}{l}\text { North } \\
\text { South }\end{array}$ & $\begin{array}{l}2-16-72 \\
2-16=72\end{array}$ & $\begin{array}{l}3-15-76 \\
3-15-76\end{array}$ & $\begin{array}{l}\text { DOP } \\
\text { DOP }\end{array}$ & $\begin{array}{l}99.996 \\
99.995\end{array}$ \\
\hline
\end{tabular}

The CWS filters in the cell ventilation system were checked in series. 
Table 10 (continued)

\begin{tabular}{|c|c|c|c|c|c|}
\hline $\begin{array}{l}\text { Type. } \\
\text { Filter }\end{array}$ & $\begin{array}{c}\text { Bank } \\
\text { Designation }\end{array}$ & $\begin{array}{l}\text { Date } \\
\text { Installed }\end{array}$ & $\begin{array}{l}\text { Date Last } \\
\text { Tested }\end{array}$ & Type Test & $\begin{array}{c}\text { Retention } \\
\text { Efficiency }(\%)\end{array}$ \\
\hline \multicolumn{6}{|c|}{ Normal off-Gas Filters } \\
\hline $\begin{array}{l}\text { CWS } \\
\text { CWS }\end{array}$ & $\begin{array}{l}\text { hest } \\
\text { East }\end{array}$ & $\begin{array}{l}2-12-76 \\
6-12-75\end{array}$ & $\begin{array}{l}3-24-76 \\
3-24-76\end{array}$ & $\begin{array}{l}\text { DOP } \\
\text { DOP }\end{array}$ & $\begin{array}{l}99.996 \\
99.998\end{array}$ \\
\hline $\begin{array}{l}\text { Sharcoal } \\
\text { Sharcoal }\end{array}$ & $\begin{array}{l}\text { Lest } \\
\text { East }\end{array}$ & $\begin{array}{l}2-12-76 \\
7-29-75\end{array}$ & $\begin{array}{l}2-17-76 \\
2-24-76\end{array}$ & $\begin{array}{l}\text { Elemental Iodine } \\
\text { Elemental Iodine }\end{array}$ & $\begin{array}{l}99.99 \\
95.76\end{array}$ \\
\hline $\begin{array}{l}\text { Charcoal } \\
\text { Charcoal }\end{array}$ & $\begin{array}{l}\text { Fiest } \\
\text { East }\end{array}$ & $\begin{array}{l}2-12-76 \\
7-29-75\end{array}$ & $\begin{array}{l}4-20-76 \\
8-27-75\end{array}$ & $\begin{array}{l}\text { Methyl Iodide } \\
\text { Methy1 Iodide }\end{array}$ & $\begin{array}{l}99.93 \\
99.41\end{array}$ \\
\hline
\end{tabular}


Table 11. Experiment Facility Assignment and Usage

\begin{tabular}{|c|c|c|c|c|c|}
\hline Facility & $\begin{array}{l}\text { Access } \\
\text { Flange }\end{array}$ & $\begin{array}{l}\text { Date } \\
\text { Installed }\end{array}$ & $\begin{array}{l}\text { Date } \\
\text { Removed }\end{array}$ & Description of Experiment & $\begin{array}{l}\text { Division or Other } \\
\text { Sponsor }\end{array}$ \\
\hline B-8 & $V-1$ & $12-10-75$ & $1-27-76$ & Pressurized Capsule & Metals and Ceramics \\
\hline B-8 & $v-1$ & $1-27-76$ & & $\begin{array}{l}\text { Four internally pressurized } \\
\mathrm{Zr} \text { capsules. }\end{array}$ & Metals and Ceramics \\
\hline $\mathrm{D}-8$ & $v-3$ & $3-10-67$ & & $\begin{array}{l}\text { Hydraulic Tube No. } 2 \\
\text { used for isotope production } \\
\text { and short term irradiations }\end{array}$ & Operations \\
\hline$E-3$ & $v-6$ & $5-6-75$ & & P-13T Capsule Experiment & Reactor \\
\hline$E-7$ & $v-5$ & $6-20-75$ & & OF-2 Capsule Experiment & Reactor \\
\hline$A-3$ & None & $8-15-75$ & . & $\begin{array}{l}\text { Radioisotopes (iodine and } \\
\text { xenon) }\end{array}$ & Isotopes \\
\hline$P-4 A$ & None & $11-3-75$ & $1-27-75$ & TeGen II Capsule Experiment & Reactor \\
\hline$P-4 A$ & None & $1-28-76$ & $3-26-75$ & TeGen III Capsule Experiment & Reactor \\
\hline$P-5$ & None & $12-10-75$ & $1-27-7 \overline{5}$ & Tensile specimens & Metals and Ceramics \\
\hline $\mathrm{P}-5$ & None & $1-27-76$ & $3-26-75$ & Tensile specimens & Metals and Ceramics \\
\hline $\mathrm{P}-7-\mathrm{P}-8$ & None & $12-30-75$ & $1-5-76$ & Nose cone experiment & General Electric \\
\hline GCR-P7-A & None & $8-8-72$ & & GB-10 Capsule experiment & Reactor \\
\hline
\end{tabular}


Table 11 (continued)

\begin{tabular}{|c|c|c|c|c|c|}
\hline Facility & $\begin{array}{l}\text { Access } \\
\text { Flange }\end{array}$ & $\begin{array}{l}\text { Date } \\
\text { Installed }\end{array}$ & $\begin{array}{l}\text { Date } \\
\text { Removed }\end{array}$ & Description of Experiment & $\begin{array}{l}\text { Division or Other } \\
\text { Sponsor }\end{array}$ \\
\hline $\mathrm{HB}-2$ & None & $7-58$ & & Weutron diffraction experiments & Solid State Physics \\
\hline $\mathrm{HB}-3$ & Wone & $11-1-58$ & & Meutron diffraction experiments & Solid State Physics \\
\hline $\mathrm{HB}-4$ & None & $7-58$ & & Meutron diffraction experiments & Chemistry \\
\hline $\mathrm{HB}-6$ & None & & & & Solid State Physics \\
\hline $\mathrm{HN}-3$ & None & $11-59$ & & Activation Analysis & Analytical Chemistry \\
\hline $\mathrm{HN}-4$ & None & $12-15-63$ & & $\begin{array}{l}\text { Void determinations in } \\
\text { irradiated materials }\end{array}$ & Solid State Physics \\
\hline $\mathrm{HN}-4$ & None & $12-15-63$ & & $\begin{array}{l}\text { "Fly's Eye" two-dimensional } \\
\text { neutron detector for neutron } \\
\text { diffraction and radiography }\end{array}$ & $\begin{array}{l}\text { Instrumentation } \\
\text { and Controls }\end{array}$ \\
\hline
\end{tabular}




\section{SPECIAL TEST}

Special measurements were made in CP-E5 during the end of Cycle 129 shutdown to test the operation of instrumentation that was designed to measure gamma heating and fission rates. The test consisted of three low power runs $\left(\mathrm{N}_{\mathrm{L}}\right)$ using special constructed calorimeters. 
ORNL/TM-5623

INTERNAL DISTRIBUTION

1. R. G. Affel

2. M. A. Baker

3. F. T. Binford

4. R. K. Branam

5. C. D. Cagle

6. W. R. Casto

7. G. H. Coleman

8. J. A. Conlin

9. J. A. Cox

10. F. L. Culler

11. S. J. Ditto

12. B. C. Duggins

13. W. A. Duggins

14. C. B. Gaither

15. E. D. Gupton

16. T. P. Hamrick

17. V. 0. Haynes

18. S. S. Hurt, III

19. L. P. Jernigan

20. E. B. Johnson

21. S. I. Kaplan

22. O. L. Keller

23. E. M. King

24. Eugene Lamb
25. E. D. Lance

26. L. C. Lasher

27. R. V. McCord

28. F. H. Neill

29. T. B. Nixon

30. L. C. Oakes

31. Herman Postma

32. M. E. Ramsey

33. J. B. Ruble

34. T. M. Sims

35. M. J. Skinner

36. J. H. Swanks

37. J. R. Thomas

38. W. E. Thomas

39. K. R. Thoms

40. J. R. Weir, Jr.

41. K. W. West

42. E. J. Witkowski

43-45. Laboratory Records Department

46. Laboratory Records, ORNL R.C.

47-48. Central Research Library

49. Document Reference Section

EXTERNAL DISTRIBUTION

50. K. M. Akhtar, Pakistan

51. Richard Brown-Campos, Puerto Rico

52-53. M. B. Biles, ERDA, Washington

54. Eric Blomber, Sweden

55. K. J. Bobin, Belgium

56. ERDA-ORO: Safety and Environmental Control Division

57. C. Moller, Republic of South Africa

58. Director; Reactor Division, ERDA-ORO

59. Kiyoshi Inoue, Hitachi, Japan

60. M. Jacquemain, France

61. Mas anori Kanbara, JAERI, Japan

62. W. Krull, West German Federal Republic

63. Research and Technical Support Division, ERDA-ORO

64. 0. D. Oner, Turkey

65. G. Rada, Venezuela

66. R. J. Swanenburg de Veye, The Netherlands

67-93. Technical Information Center, Oak Ridge

94. R. Wakabayashi, NAIG, Japan

95. K. E. Wirtz, West German Federal Republic

96. A. C. Woods, Australia 